$\mathbf{m} / \mathbf{s}$

médecine/sciences $85 ; 1: 292-3$

\title{
L'ENDOCRINOLOGIE EN MUTATION
}

\section{Xavier Bertagna \\ Professeur à la faculté Cochin Port-Royal, Université Paris- $V$. Clinique des maladies endocriniennes et méta- boliques, hôpital Cochin.}

\section{ADRESSE}

Hôpital Cochin, 27, rue du Faubourg-Saint-Jacques, 75014 Paris.

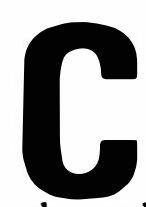

hoisir trois thèmes pour illustrer les progrès les plus récents de l'endocrinologie moderne est presque une gageure quand on mesure l'extraordinaire diversité d'une spécialité qui étend chaque jour le champ de ses applications. Deux articles publiés ci-après, auxquels s'ajoutera un troisième dans un de nos prochains numéros, sont chacun révélateur d'un mode de progrès en endocrinologie, puisqu'on y verra comment découvrir, manipuler et même créer les hormones des années 90.

Plus que jamais, la découverte de nouvelles hormones est une activité en pleine expansion. Dernière en date, celle du facteur natriurétique de l'oreillette confirme avec éclat une hypothèse ancienne : le cœur a ses hormones que la raison subodorait [I]. L'article de M. Cantin, p. 294, raconte l'histoire de cette découverte et fait ressortir toutes ses implications. Cet article est démonstratif d'une certaine démarche endocrinologique qui mêle, à la fois, le classique et le moderne : classique, le repérage dans un organe d'une activité biologique (natriurétique et diurétique) et l'extraction à partir de cet organe de la molécule responsable; moderne, l'apport de la génétique moléculaire [2] qui permet d'établir immédiatement la structure du précurseur peptidique, l'organisation et la localisation chromosomique du gène.

En matière de pharmacologie endocrinienne, la manipulation de la fonction gonadotrope par la LHRH (luteinizing hormone releasing hormone) et ses agonistes est un autre exemple des progrès de l'endocrinologie moderne (voir rubrique hypothèses et débats, p. 32I). Isolé en $197 \mathrm{I}$, ce peptide hypothalamique est le principal facteur de régulation des hormones gonadotropes [3]. Le fait majeur révélé par les travaux de Knobil [4] est que, selon son mode d'administration, continu ou au contraire discontinu par "pulses ", la LHRH exerce des effets exactement opposés : dans le premier cas elle supprime, dans le deuxième cas elle stimule la fonction gonadotrope. Outre l'importance de ce phénomène, sur un plan purement fondamental, il y avait là un concept pharmacologique tout à fait original et bien évidemment l'espoir de nouvelles actions thérapeutiques modulées en fonction des modalités d'administration de la molécule. Ces actions thérapeutiques sont maintenant largement utilisées comme le rapporte l'article de $F$. Labrie, $p$. 300 : ainsi l'administration discontinue de LHRH permet de rétablir la fertilité par induction de l'ovulation ou de la spermatogenèse dans

\section{REFEERENCES}

I. Sagnella GA, MacGregor GA Cardiac peptides ant the control of sodium excretion. Nature 1984; 309 : 666-7.

2. Nemer $M$, Chamberland $M$, Sinois D, et al. Gene structure of human cardiac hormone precursor, pronatriodilatin. Nature 1984; $312: 654-6$.

3. Guillemin R. Peptides in the brain: the new endocrinology of the neuron. Science 1978; 202 : 390-402.

4. Knobil E. The neuroendocrine control of the menstrual cycle. Rec Progr Horm Res 1980; $35:$ 53-88.

5. Hughes J, Smith TW, Kosterlitz HW, Fothergill LA, Morgan BA, Morris HR. Identification of two related pentapeptides from the brain with potent opiate agonist activity. Nature 1975; 258 : 577-9.

6. Schwartz JC, Roques BP. Opioid peptides as intercellular messengers. Biomedicine 1980; 32 : 169-75.

7. Robinson AG. DDAVP in the treatment of central diabetes insipidus. N Engl 7 Med 1976; 294 : 507-II.

8. Shibahara S, Morimoto $Y$, Furatani $Y$, et al. Isolation and sequence analysis of the human corticotropin releasing factor precursor gene. Embo 7 1983; 2 : 775-9.

9. Barritault D, Moenner $M$, Loret C. Nature et propriétés de divers facteurs de croissance. médecine/sciences $1985 ; 1$ : 80-5. 
certains dysfonctionnements gonadotropes. A l'inverse, l'administration continue de LHRH permet le traitement des pubertés précoces, de l'endométriose, du cancer du sein, et sera probablement utilisé comme moyen contraceptif. F. Labrie met également l'accent sur le remarquable progrès thérapeutique que constitue l'utilisation des agonistes de la LHRH dans le traitement du cancer de la prostate où, par leur effet anti-gonadotrope, ils permettent la réalisation d'une véritable " castration chimique ".

La création de nouvelles molécules est un art ancien. Il tend aujourd'hui à devenir une véritable science, comme le démontrera l'article de B. P. Roques, dans le domaine particulier de la chimie des peptides (à paraître en janvier 86 dans médecine/sciences). L'importance de ces nouvelles techniques (volontiers appelées drug design) n'est nulle part ailleurs mieux illustrée que dans le champ de la neuro-endocrinologie. Ici la synthèse de molécules antagonistes est indispensable pour comprendre la signification physiologique de certains systèmes neuronaux, puisque l'absence de délimitation anatomique nette interdit l'utilisation des méthodes anciennes (ablation de l'organe). Par exemple, la naloxone, antagoniste de la morphine, a été l'outil pharmacologique indispensable pour isoler les peptides opioïdes [5].

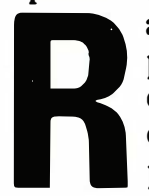
apidement, à partir des premières structures peptidiques caractérisées (les enképhalines), le chimiste a pu ensuite synthétiser toute une série d'analogues d'action soit agoniste, soit antagoniste, qui ont permis la caractérisation des différents récepteurs opioïdes [6]. De la même façon, on peut, à partir d'un modèle, créer des variantes pour modifier l'expression biologique d'un peptide et améliorer ses performances thérapeutiques.

L'exemple du DDAVP (Minirin) analogue de la vasopressine (voir rubrique hypothèses et débats, p. 32I) est à cet égard particulièrement démonstratif [7] : en modifiant deux acides aminés de l'octapeptide naturel, les chimistes ont d'une part complètement dissocié les activités antidiurétique et pressive (au profit de la première) et d'autre part, considérablement ralenti le métabolisme du peptide. On obtient ainsi un analogue puissant, d'action prolongée après administration par voie nasale et dénué d'effet secondaire, ce qui constitue un progrès considérable pour les patients atteints de diabète insipide. Cet exemple, comme celui des inhibiteurs de l'enzyme de conversion dans le domaine de l'hypertension artérielle, pourrait contribuer à changer l'attitude de l'industrie pharmaceutique, souvent réticente vis-à-vis des médicaments peptidiques, en raison des difficultés de leur maniement. La génération des " pseudo-peptides " devrait rapidement prendre le relai et faciliter cette conversion.

Au-delà de leur intérêt ponctuel, les trois thèmes retenus sont révélateurs de deux phénomènes étroitement liés à l'évolution de l'endocrinologie moderne : le premier tient à la part de plus en plus grande prise par les peptides en endocrinologie. Ce type de molécule constitue l'énorme majorité des hormones nouvellement découvertes. Comme souvent, les progrès de la technologie ont été les éléments contributifs essentiels. Nous le verrons pour ce qui concerne la chimie des peptides dans l'article de B. P. Roques, à paraître. Aussi importante a été la percée technologique de la génétique moléculaire, dont le pouvoir amplificateur a radicalement modifié l'ordre de grandeur des problèmes. Par exemple, la purification des premiers peptides hypothalamiques avait nécessité le recueil de centaines de milliers d'hypothalamus de mouton et de porc; aujourd'hui le CRF humain (corticotropin releasing factor) a été caractérisé sans mềme avoir été purifié, grâce à une sonde d'ADN permettant l'analyse directe du gène [8] à partir d'une banque génomique humaine. Le deuxième phénomène, également illustré ici, est celui de la diversité endocrinienne. Celle-ci a certes toujours existé, puisqu'aucun organe n'est insensible à l'action des hormones; ces dernières cependant restaient les produits d'un nombre réduit d'organes parfaitement délimités anatomiquement et l'endocrinologue pouvait, à juste titre, s'intituler le médecin des "glandes" endocrines. Aujourd'hui, l'endocrinologie atteint un niveau de diversité d'un tout autre ordre : si tous les organes sont concernés par les hormones, c'est aussi parce que tous produisent des hormones : le rein, le poumon, le tube digestif, le cerveau... et maintenant le cœur, sont autant de nouveaux producteurs. Dans ces organes, les sites de production sont en général anatomiquement mal définis, sans regroupement cellulaire net (réseaux neuronaux, cellules dispersées). A cette diversité anatomique, s'ajoute celle des modes de production hormonale: sécrétion endocrine classique mais également neurosécrétion, neurotransmission, neuromodulation, sécrétion paracrine ou autocrine [9].

inalement, toutes ces
variantes sont reconnues
comme autant de modes
d'expression différents
d'une même activité de
type endocrine. Le champ classique de l'endocrinologie s'en trouve considérablement élargi. Ainsi, par exemple, il s'étend à l'important domaine des facteurs de croissance dont on sait l'implication en cancérologie, et en toute logique également, à celui de l'immunologie. Dans ce cas, des cellules immunocompétentes peuvent répondre à une stimulation en sécrétant des substances qui agissent à distance, telles les lymphokines, selon un processus qui s'apparente à une activité de type " endocrine".

Par son évolution récente, l'endocrinologie moderne atteint donc un niveau de diversité qui rend indispensable la redéfinition d'une spécialité véritablement... éclatée. A la conception classique qui organisait l'endocrinologie autour des grands pôles représentés par les "glandes endocrines", devrait se substituer celle d'un nouvel espace endocrinien regroupant des "systèmes hormonaux " définis selon leurs caractéristiques fonctionnelles et non anatomiques, par leur interaction dans chacune des grandes fonctions de l'organisme 